\title{
Fluorescence Mapping of PCP Light-Harvesting Complexes Coupled to Silver Nanowires
}

\author{
B. Krajnik ${ }^{a, *}$, D. Piatkowski ${ }^{a}$, M. Olejnik ${ }^{a}$, N. Czechowski ${ }^{a}$, E. Hofmann $^{b}$, \\ W. HEISS ${ }^{c}$ AND S. MACKOWSKI ${ }^{a}$ \\ ${ }^{a}$ Institute of Physics, Nicolaus Copernicus University, Grudziądzka 5, 87-100 Toruń, Poland \\ ${ }^{b}$ Department of Biology and Biotechnology, Ruhr-University Bochum, Bochum, Germany \\ ${ }^{c}$ Institute of Semiconductor and Solid State Physics, Johannes Kepler University, Linz, Austria
}

\begin{abstract}
In this work we demonstrate confocal fluorescence imaging of hybrid nanostructures composed of silver nanowires and peridinin-chlorophyll-protein light-harvesting complexes. The length of silver nanowires, which reaches $10 \mu \mathrm{m}$, allows for determination of the nanowire position and consequently direct correlation with the fluorescence image. In this way we probe the influence of plasmon induced electromagnetic field on the fluorescence of light-harvesting complexes. When the nanowires are spaced from the light-harvesting complexes by a $10 \mathrm{~nm}$ thick dielectric layer, we observe a fluorescence enhancement, which depends upon the laser excitation wavelength. The measured enhancement values are 2.5 and 1.9 for $405 \mathrm{~nm}$ and $485 \mathrm{~nm}$, respectively. Larger enhancement for the $405 \mathrm{~nm}$ excitation is attributed to direct creation of plasmon excitations in the silver nanowires.
\end{abstract}

PACS: 87.64.kv, 42.62.Fi, 81.07.Pr, 87.64.M-

\section{Introduction}

Electromagnetic field enhancement generated by plasmon oscillations in metallic nanoparticles has been shown to influence both absorption and emission of nearby placed emitters [1-4]. Therefore, developing ways to manipulate plasmon resonances has been important for applying these nanostructures in biosensors, bioimaging, and various tip-enhanced spectroscopies. Recently, attempts have been made to couple complex pigmentprotein systems with metallic nanoparticles in order to investigate possibilities to enhance the absorption of the photosynthetic complexes [5-8]. These research efforts have been so far focused on spherical nanoparticles, with plasmon resonances between $420 \mathrm{~nm}$ and $550 \mathrm{~nm}$. Extending the pool of metallic nanoparticles coupled to the light-harvesting complexes is important for optimizing the effect of plasmon enhancement.

Due to diffraction-imposed limitations, observation of plasmon induced effects on a single nanoparticle level is hindered and limited to nanoparticles with one dimension reaching $\mu \mathrm{m}$ sizes. An example of such a metallic nanostructure is a silver nanowire, with diameters in the range of 100-200 $\mathrm{nm}$ and lenghts of 5-10 $\mu \mathrm{m}$ [9]. Preliminary results indicate that fluorescence imaging of such nanostructures is possible and indeed direct deposition of emitting molecules leads to fluorescence en-

\footnotetext{
* corresponding author; e-mail: bakrag@fizyka.umk.pl
}

hancement [10]. However, direct deposition of light-harvesting complexes on metallic substrates results in at least partial quenching of the fluorescence due to efficient non-radiative transfer to metallic nanoparticles [4]. In this work we focus on a hybrid nanostructure where the nanowires are covered by a thin, $10 \mathrm{~nm}$ layer of $\mathrm{SiO}_{2}$ in order to inhibit the quenching. Fluorescence imaging of such a system demonstrates strong enhancement of fluorescence of peridinin-chlorophyll-protein complexes. While the emission spectrum is not affected, shape- and energy-wise by the proximity of metallic nanoparticles, the enhancement shows substantial dependence upon the wavelength of laser excitation. When the laser is tuned directly to the maximum of plasmon resonance in the silver nanowire $(405 \mathrm{~nm})$, the enhancement factor of 2.5 is reached. For off-resonance excitation (485 nm) the enhancement factor is $30 \%$ lower. We believe that these results are important for understanding the plasmon enhanced absorption in light-harvesting complexes and determining the optimal conditions for applying hybrid nanostructures involving photosynthetic proteins for applications in photovoltaics devices and optoelectronics.

\section{Materials and methods}

Water-soluble peridinin-chlorophyll-protein (PCP) light-harvesting complex is a peripheral antenna from dinoflagellate Amphidinium carterae. The major function of this complex is to harvest sunlight and transfer the energy subsequently to the reaction center for next steps 
of photosynthesis. The PCP is in a way unique among light-harvesting complexes, as it utilizes carotenoid, i.e. peridinin, as its main light-harvesting complex, not the chlorophylls.

The structure of this complex, determined using X-ray crystallography with a resolution of $2.0 \AA$ [11], is shown in Fig. 1a. The PCP is a relatively small protein-pigment complex (weight of $32 \mathrm{kDa}$ ), in which pigments are organized in two clusters (halfmers). Each halfmer contains four peridinins (orange), one chlorophyll $a$ (green) and one lipid molecule (blue). The distance between chlorophylls $a(\mathrm{Chl} a)$ in a monomer is $17.4 \AA$. Spectroscopic properties of the PCP complexes have been extensively studied on the ensemble [12-14] and single-molecule level [15-17] providing a frame to understand the energy transfer pathways in this system. In this work we use PCP complexes obtained by reconstitution of $N$-terminal apoprotein domains extracted from modified Escherichia coli with appropriate pigments [18]. The final product was purified by passage through a PD10 and DEAE tris-acryl column and equilibrated with TRIS buffer (pH 7.6).
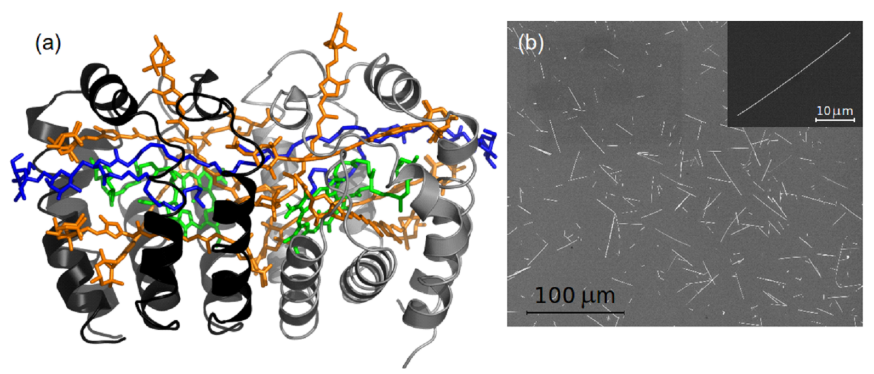

Fig. 1. (a) Structure of a monomer of reconstituted PCP complex: four peridinins, one chlorophyll $a$ and one lipid molecule. PDB entry 3IIS. (b) Scanning electron microscopy image of silver nanowires.

Silver nanowires were synthesized using polyol process in which the ethylene glycol served as the reducing and solvent reagent [9]. This method gives us an opportunity to carry out reaction in the soft condition with temperatures $<200^{\circ} \mathrm{C}$ and atmospheric pressure.

Silver nitrate is reduced by ethylene glycol in the presence of poly(vinylpyrrolidone) (PVP). PVP acts as a stabilizing agent - it creates a protective coating onto the silver surface thus preventing from aggregation of silver nanowires. In typical synthesis, $40 \mu \mathrm{l}$ of $\mathrm{CuCl}_{2}$ solution ( $4 \mathrm{mM}$ in $\mathrm{EG}$ ) was added to $5 \mathrm{~mL}$ of ethylene glycol in glass vial, heated at $150^{\circ} \mathrm{C}$ with stirring. After $15 \mathrm{~min}$, $1.5 \mathrm{~mL}$ of PVP solution (114 mM in EG) and $1.5 \mathrm{~mL}$ $\mathrm{AgNO}_{3}$ solution (94 mM in EG) was then simultaneously added dropwise using a syringe pump. The reaction mixture was then heated and kept hot for one hour. The product was purified by centrifugation at $2000 \mathrm{rpm}$ for 20 min and redispersed in pure water.

In Fig. 1b we show the scanning microscopy image of the silver nanowires. The estimated length of the nanowires varies from few to few tens of microns with typical diameters about $200 \mathrm{~nm}$. The sample was prepared by spin-coating first the solution of silver nanowires on a substrate. Then, in order to assure for separation between light-harvesting complexes and metallic nanoparticles an $\mathrm{SiO}_{2}$ spacer with $10 \mathrm{~nm}$ thickness was deposited using e-beam assisted evaporation technique. On the top of the substrate $10 \mu \mathrm{L}$ of PCP diluted in $2 \%$ PVA water solution was dropped and spin-coated for $60 \mathrm{~s}$ with speed of $2100 \mathrm{rpm}$.

Fluorescence intensity and emission spectra measurements were performed on our home-built fluorescence confocal microscope based on Olympus infinity-corrected microscope objective LMPlan 50x (NA 0.5) [19]. The sample is placed on a piezoelectric stage (Physik Instrumente), which enables us to collect fluorescence intensity maps by combining fluorescence intensity measurements with the motion of the $X Y$ translation stage. Fluorescence intensity maps are collected with an avalanche photodiode (Perkin-Elmer SPCM-AQRH-14). This experimental configuration allows for measuring fluorescence intensity and emission spectra. The emission is dispersed using Amici prism and detected with a CCD camera (Andor iDus DV 420A-BV). For imaging the position of the nanowires in a reflection mode we use a $598 \mathrm{~nm}$ laser, which is practically not absorbed by the PCP complex. The fluorescence on the other hand was excited with either $405 \mathrm{~nm}$ or $485 \mathrm{~nm}$ laser. The excitation power used in all experiments for each laser was about $60 \mu \mathrm{W}$.

\section{Results and discussion}

Absorption and emission spectra of the PCP complex reconstituted with Chl $a$ are shown in Fig. 2a. Peridinin (Per) is responsible for a dominant, blue-green absorption band from $350 \mathrm{~nm}$ to $550 \mathrm{~nm}$, while Chl a absorbs at $668 \mathrm{~nm}\left(Q_{y}\right.$ band) and $440 \mathrm{~nm}$ (Soret band). The emission of the PCP complexes originates from $Q_{y}$ transition of Chl $a$ and is located at $673 \mathrm{~nm}$.
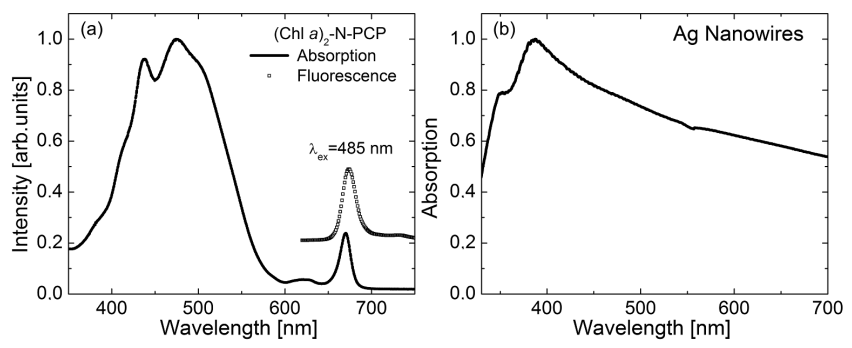

Fig. 2. (a) Absorption and fluorescence spectrum of the PCP complexes measured at room temperature. The excitation wavelength for the fluorescence was $485 \mathrm{~nm}$. (b) Absorption spectrum of silver nanowires in water solution.

The absorption spectrum of silver nanowires in aqueous solution has a maximum at $380 \mathrm{~nm}$ and is shown in Fig. 2b. The large Stokes shift between strong absorption 
and fluorescence of the PCP (over $100 \mathrm{~nm}$ ) and significant spectral overlap between absorption of Per and silver nanowires renders PCP a model system for studying plasmonic interactions in such hybrid nanostructures.

In Fig. 3 we show examples of images acquired for a hybrid nanostructure composed of silver nanowires and PCP complexes. All the images are $20 \mu \mathrm{m} \times 20 \mu \mathrm{m}$ in size. During the experiment we first aim at finding an area on the sample surface with an individual isolated nanowire defined. For that purpose a $589 \mathrm{~nm}$ laser was used with a bandpass filter HQ 610/40 (Chroma) coupled to the detector. Since the PCP absorption at this wavelength is negligible, photophysics of the complexes is not affected by the laser radiation.
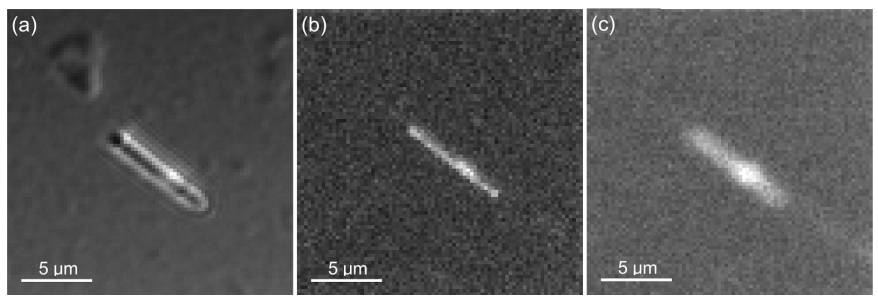

Fig. 3. Intensity maps obtained for a hybrid nanostructure built of silver nanowires and PCP complexes. (a) Reflection image of silver nanowires, excitation wavelength $589 \mathrm{~nm}$ with $610 / 40$ bandpass filter for detection. (b) Fluorescence image of PCP complexes, excitation wavelength $405 \mathrm{~nm}$ with $670 / 10$ bandpass filter for detection. (c) Fluorescence image of PCP complexes, excitation wavelength $485 \mathrm{~nm}$ with $670 / 10$ bandpass filter for detection.

Upon selecting a proper area in a reflection mode (Fig. 3a) we switched the excitation to laser wavelengths, which are efficiently absorbed by Per in the PCP complex.

The fluorescence images for $405 \mathrm{~nm}$ and $485 \mathrm{~nm}$ taken from the same area are shown in Fig. $3 \mathrm{~b}$ and c, respectively. In this case the emission of PCP complexes was extracted with HQ 670/10 (Chroma) bandpass filter. For both excitation lasers the fluorescence intensity for PCP complexes deposited directly on the silver nanowire is significantly higher than for PCP complexes located away from the nanowire.

In order to quantitatively analyze the fluorescence maps and the enhancement of the emission intensity due to the coupling with plasmon excitations in the silver nanowires we determine intensity cross-sections along the long axis of the nanowire. The intensity changes over the nanowire for both excitation wavelengths are shown in Fig. 4. In the case of $405 \mathrm{~nm}$ excitation wavelength the average intensity on the nanowire $117 \mathrm{cps}$, while off the nanowire we obtain $47 \mathrm{cps}$. Corresponding values for the $485 \mathrm{~nm}$ excitation are $398 \mathrm{cps}$ and $203 \mathrm{cps}$, respectively. Therefore, the average fluorescence enhancement factors are 2.49 and 1.96 for $405 \mathrm{~nm}$ and $485 \mathrm{~nm}$ excitation, respectively. This result is in agreement with the absorption spectrum of the silver nanowires, the inten- sity measured at $405 \mathrm{~nm}$ is approximately $25 \%$ higher than that at $485 \mathrm{~nm}$. The dependence of the enhancement upon the excitation wavelength points clearly at plasmonic origin of this effect. Such an analysis is based on the assumption that the laser induced photobleaching is negligible and that it depends weakly on whether the PCP complexes are on or off the nanowires. This assumption is justified with extremely low excitation power of the lasers used for measuring the fluorescence of the light-harvesting complexes.

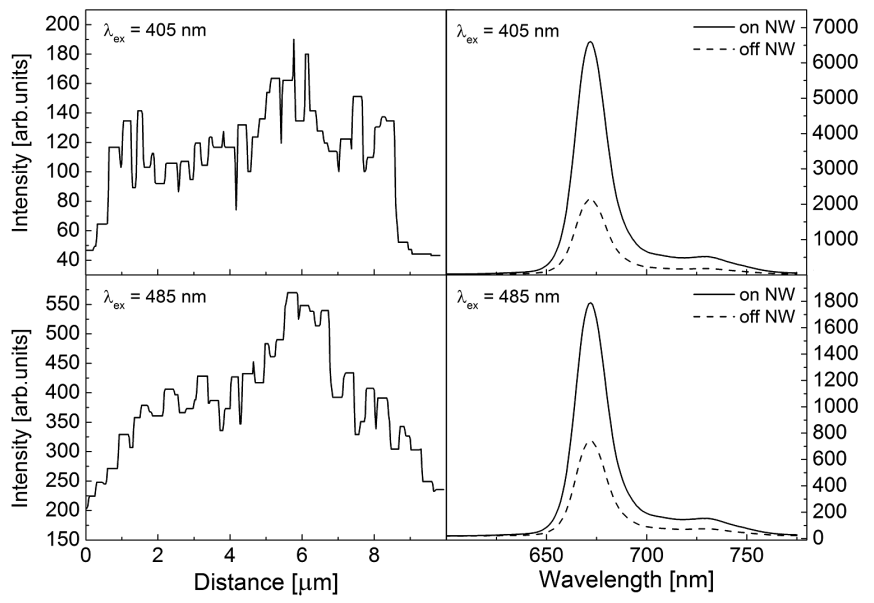

Fig. 4. Cross-sections of fluorescence intensity of the PCP complexes obtained along the nanowire for $405 \mathrm{~nm}$ (upper left) and $485 \mathrm{~nm}$ (bottom left) excitation wavelength. Emission spectra measured on (solid line) and off (dotted line) a single nanowire for $405 \mathrm{~nm}$ (upper right) and $485 \mathrm{~nm}$ (bottom right) excitation wavelengths.

Fluorescence spectra of PCP complexes obtained for both excitations wavelengths are shown in Fig. 4. We compare typical spectra measured off the nanowire (dashed line) and on the nanowire (solid line). For both excitation wavelengths the shapes of the emission are identical, the only difference is associated with the intensity. This observation implies, similarly to the case of PCP complexes deposited on the silver island film [10], that the protein remains intact upon coupling to the metallic nanoparticles.

\section{Conclusions}

Fluorescence imaging of hybrid nanostructures composed of peridinin-chlorophyll-protein complex deposited on individual silver nanowires demonstrates that the emission of the chlorophylls in this light-harvesting complex is enhanced due to plasmon interactions. The enhancement depends on the excitation wavelength, thus the efficiency of plasmon excitations: for optimal excitation conditions, which correspond to $405 \mathrm{~nm}$, the enhancement factor is approximately $25 \%$ larger than for $485 \mathrm{~nm}$ excitation wavelength. Importantly, the functionality of the light-harvesting complex is not ham- 
pered in any way due to the proximity of metallic nanostructures. The results show great potential for manipulating the optical properties of complex biomolecules using plasmon excitations in metallic nanoparticles.

\section{Acknowledgments}

Financial support from the WELCOME program "Hybrid nanostructures as a stepping-stone towards efficient artificial photosynthesis" awarded by the Foundation for Polish Science is acknowledged.

\section{References}

[1] P. Anger, P. Bharadwaj, L. Novotny, Phys. Rev. Lett. 96, $113002(2006)$.

[2] J. Lee, T. Javed, T. Skeini, A.O. Govorov, G.W. Bryant, N.A. Kotov, Angew. Chem. 45, 4819 (2006).

[3] K. Ray, R. Badugu, J.R. Lakowicz, J. Am. Chem. Soc. 128, 8998 (2006).

[4] S. Mackowski, S. Wormke, A.J. Maier, T.H.P. Brotosudarmo, H. Harutyunyan, A. Hartschuh, A.O. Govorov, H. Scheer, Ch. Brauchle, Nano Lett. 8, 558 (2008).

[5] J. Nieder, R. Bittl, M. Brecht, Angew. Chem. 49, 10217 (2010).

[6] I. Carmeli, I. Lieberman, L. Kraversky, Z. Fan, A.O. Govorov, G. Markovich, S. Richter, Nano Lett. 10, 2069 (2010).

[7] L. Bujak, N. Czechowski, D. Piatkowski, R. Litvin, S. Mackowski, T.H.P. Brotosudarmo, R.J. Cogdel, S. Pichler. W. Heiss, Appl. Phys. Lett. 99, 173701 (2011).

[8] N. Czechowski, P. Nyga, M.K. Schmidt, T.H.P. Brotosudarmo, H. Scheer, D. Piatkowski, S. Mackowski, Plasmonics 7, 115 (2012).
[9] Y. Sun, Y. Yin, B.T. Mayers, T. Herricks, Y. Xia, Chem. Mater. 14, 4736 (2002).

[10] L. Bujak, D. Piatkowski, S. Mackowski, S. Wormke, C. Jung, C. Brauchle, A. Agarwal, N.A. Kotov, T. Schulte, E. Hofmann, T.H.P. Brotosudarmo, H. Scheer, A.O. Govorov, R.G. Hiller, Acta Phys. Pol. A 116, S-22 (2009).

[11] E. Hofmann, P.M. Wrench, F.P. Sharples, R.G. Hiller, W. Welte, K. Diederichs, Science 272, 5269 (1996).

[12] F.J. Kleima, M. Wendling, E. Hofmann, E.J.G. Peterman, R. van Grondelle, H. van Amerongen, Biophys. J. 78, 344 (2000).

[13] T. Polivka, V. Sundstrom, Chem. Rev. 104, 2021 (2004).

[14] T. Schulte, S. Johanning, E. Hofmann, Proc. Natl. Acad. Sci. USA 106, 20764 (2010).

[15] S. Wormke, S. Mackowski, T.H.P. Brotosudarmo, C. Jung, A. Zumbusch, M. Ehrl, H. Scheer, E. Hofmann, R.G. Hiller, C. Brauchle, Biochim. Biophys. Acta 1767, 956 (2007)

[16] S. Mackowski, S. Wormke, T.H.P. Brotosudarmo, C. Jung, R.G. Hiller, H. Scheer, C. Brauchle, Biophys. J. 93, 3249 (2007).

[17] S. Mackowski, S. Wormke, T H.P. Brotosudarmo, H. Scheer, C. Brauchle, Photosynth. Res. 95, 253 (2008).

[18] T.H.P. Brotosudarmo, E. Hofmann, R.G. Hiller, S. Wormke, S. Mackowski, A. Zumbusch, C. Brauchle, H. Scheer, FEBS Lett. 580, 5257 (2006).

[19] B. Krajnik, T. Schulte, D. Piątkowski, N. Czechowski, E. Hofmann, S. Mackowski, Cent. Eur. J. Phys. 2, 293 (2011). 